\title{
Guilt and Shame Proneness in Relations to Covert Narcissism among Emerging Adults
}

\author{
Mahak Mathur, Priyanshi Khandelwal*, Sakshi \\ Jain, Vedika Jain \\ Department of Psychology, Mahatma Gandhi Medical College \& \\ Hospital, Jaipur, Rajasthan, India \\ Study Area:Jaipur, India \\ Coordinates: $11^{\circ} 1^{\prime} 6^{\prime \prime} \mathrm{N} ; 76^{\circ} 58^{\prime} 21$ "E
}

Key words: Vulnerable Narcissism

\section{Abstract}

It was hypothesized that there would be a significant relationship between Covert Narcissism and Shame and Guilt Proneness. Also, Guilt and Shame proneness would predict Covert Narcissism. This study comprised 180 emerging adults, in the age group of 18 to 23 years. The measure of the study included the Maladaptive Covert Narcissism and Guilt and Shame Proneness Scale. The study also investigated gender differences in covert narcissism. The results of the study indicated that there was a positive correlation between covert narcissism and the two dimensions of shame proneness namely negative selfevaluation and shame withdrawal. Additionally, no significant relationship was found between guiltproneness and covert narcissism. The results further revealed that Shame Withdrawal, a single variable regression test was used. The findings suggested that Shame Withdrawal contribute $9 \%$ variance in Covert Narcissism which is statistical significance and negative self-evaluation also significantly predicted covert narcissism accounting. Furthermore, the average score of men on covert narcissism was significantly more than that of females which means males have significantly higher tendencies to develop covert narcissism.

Overt narcissism (i.e., Grandiosity-Exhibitionism) and covert narcissism (i.e., Vulnerability-Sensitivity) are the two types of dysfunctional characteristics or maladaptive forms of narcissism (Wink, 1991). The existing information on narcissistic personality disorder focuses on various aspects of grandiose narcissism but very little has been explored on covert narcissism although both kinds share similar features such as conceit, disinterest in others, impulsiveness, risk-taking, and self-indulgence.

Some of the dissimilarities between both the forms of narcissism areas follows:

\begin{tabular}{ll}
\hline Grandiose/Overt Narcissism & Vulnerable/Covert Narcissism \\
\hline Pincus and Roche (2011) & Pincus and Roche (2011) \\
described grandiose narcissism described a socially avoidant \\
as "deeply felt desires for style of self-regulation as \\
recognition and appreciation "increased sensitivity to ego- \\
giving rise to urgent reasons to threat and subsequent self-, \\
seek out self-enhancement emotion-, and behavioural- \\
experiences". & dysregulation.
\end{tabular}

They are confident, outgoing, They are socially inhibited, and charming, but are also vain, insecure, defensive, and

*Corresponding Author:priya.khandelwal455@gmail.com 
manipulative, and aggressive ( vindictive (Hendin \& Cheek, Wink, 1991). 1997; Wink, 1991)

They have an inflated sense of They experience heightened self, viewing themselves as negative emotional reactivity, superior to others (Krizan \& including envy, shame, anxiety, Bushman, 2011), overestimating depression, and low self-esteem their intelligence and cognitive (Besser \& Priel, 2010; Wink, ability and preferring the 1991).

company of powerful and popular people (Campbell \& Foster, 2002).

They are people who grant They are the ones who grant themselves both status and self- themselves status but not love. love.

They display substantial and They are consistently consistent correlations with associated with introversion extraversion (Wink, 1991). (Wink, 1991).

Vulnerable/covert narcissism is defined by social psychology as "a defensive and insecure grandiosity that obscures feelings of inadequacy, incompetence, and negative affect" (Miller et al., 2012). Over the years, this second subtype of narcissism has gone by many names, including covert, closet, shy, or vulnerable narcissism. Vulnerable narcissists are unclear about their internal experiences, such as their self-evaluations, attitudes, and beliefs leading them to extensively rely on feedback from others (Kealy \& Rasmussen, 2012). The paradox of vulnerable narcissism is this: They believe "I deserve more!" because of their sense of entitlement, yet they are sceptical. Their lofty ambitions aren't matched by a belief in their own power. Because of this avoidance incentive, susceptible narcissists presented modestly and avoid numerous situations where their self-beliefs might be challenged (Foster \& Trimm, 20o8). As a result of skill limitations in attaining the outcomes they believe they deserve, as well as the discrepancy between internal emotions of entitlement and external modesty and shyness, identifying a susceptible narcissist can be challenging (Miller et al., 2011; Wink, 1991). Even though identifying a vulnerable narcissist and the problems he is feeling may be difficult for an outsider, the psychological stress vulnerable narcissists face is real. hypersensitive, apprehensive, timid, and insecure; yet, close interaction reveals their grandiose fantasies, which contradict those initial perceptions (Wink, 1991). Grandiose imagination, self-sacrificing selfimprovement, contingent self-esteem, and uniqueness are all signs of intrapersonal covert narcissism. Devaluing others, entitled fury, and hiding the ego are all signs of interpersonal covert narcissism. Interpersonally impaired narcissism is the phenotype of covert narcissism. These individuals have low self-esteem (Rose, 2002), high shame (Cain et al., 2008), and internalising symptoms (Krizan \& Johar, 2012). Shame also intermediates the relationship between vulnerable narcissism and a more negative model of others (Schie et al., 2021). A positive correlation was reported between covert narcissism and shame (Ghim et al., 2015). Participants with NPD had a higher level of shame than non-clinical controls, according to the literature. They also identified a link between covert narcissism and shame (Ritter, 2014).

Shame is another emergent consequence of the innate human desire to be perceived as attractive (Gilbert,1997) Internalized Shame as 'shame bound personality' or 'shame-based identity' (Kaufman, 1989). High-shame individuals have been categorized in three ways: i) more shame-prone than others in regularly shame-eliciting situations; ii) frequently or constantly experiencing generalized or global shame, also known as internalized shame, or; iii) particularly ashamed of some aspect of their behaviour or personal qualities (Andrews,1998). Shameprone people feel angrier, are more likely to interpret the actions of others negatively, and express anger in more destructive ways than primarily guilty people. Shameproneness was found to be substantially linked to psychological maladjustment. Gramzow \& Tanqney (1992) revealed that proneness to shame was negatively correlated with narcissism, a positive association between shame and narcissism emerged when maladaptive factors were isolated and shame proneness was also positively correlated with splitting, a pathological narcissistic defence. Toxic shame is a feeling that you're worthless. It happens when other people treat you poorly and you turn that treatment into a belief about yourself. Schurman (2000) found that people suffering from social anxiety and social phobia exhibit both high levels of shame and behaviour characteristic of the hypersensitive type of narcissism and there is a positive relationship between social phobia and shame. Shame is an emotion that is understudied because it is so clearly absorbed by guilt in our society (Levin, 1967). Shame is an emotion that is understudied because it is so clearly absorbed by guilt in our society (Levin, 1967). There are a variety of causes for shame's neglect, but Sigmund Freud's emphasis on guilt in his structural theory (https://blogs.uoregon.edu/autismhistoryproject/archive /sigmund-freud-on-narcissism-1914) had a key impact. Piers \& Singer (1953) were the first to establish a clear concept of shame within the psychoanalytic approach. According to Adler (1918) shame has both an isolating and an interfacing effect, emphasizing its immense importance in human civilization. Shame is a byproduct of human connectedness, and as such, it is impossible to exclude it from the human soul's life. Without this effect, human society would be unthinkable. Another factor that the present study investigates is guilt-proneness. Guilt is a cognitive or an emotional experience that occurs when an individual comprehends or believes that one has comprised one's own standards of conduct or has violated a moral standard and bears significant responsibility for that violation. It is closely related to the notion of shame, 
TECHNOSCIENCE ARTICLE

remorse, and regret. Guilt proneness is the unreasonable guilt that an individual experiences for matters which are past one's control. It is normally the legacy of a demanding childhood because of which psychologists name it selfreferencing. People who have unresolved guilt may be unpleasant or strained all the time and could be clingy or apologetic to an unhealthy degree. The feelings of guilt are frequently manifested as bodily symptoms such as insomnia or trouble sleeping, an upset stomach, nausea, muscle tension, head pain and tearfulness. Research connecting psychology and guilt do not agree always as some say that guilt may help protect people from emotional distress whereas other suggests that guilt, especially excessive guilt, adds to mental and emotional distress. According to Cohen (2012), Guilt proneness is a personality attribute indicative of a susceptibility to experiencing negative feelings about personal wrongdoing, even when the wrongdoing is private. It is identified by the apprehension of feeling bad about committing transgressions rather than by guilty feelings in a particular moment or generalized guilty feelings that occur without an eliciting event. A guilt-prone person would strive to deal with a problem that they've caused and undo the harm to others or avoid committing another transgression.

Some researchers also observed that there were negative relations between narcissism and guilt and also talked about the role played by these factors in males and females. One of the researches revealed that shame plays an important aspect in both narcissism as well as depression and that its role in depression is stronger than that of guilt. It also observed that men are more prone to narcissism and women to depression, and sex differences associated with shame and guilt are linked to self-image and specific situations (Wright et al., 1989). High Guilt Proneness trait guides the behaviour of people with high consciousness and makes them think twice before any action. According to a study done by Harder \& Greenwald (1999) revealed that shame was expected to show stronger relationships than guilt with openness, sensation seeking and extraversion. So far only a few research have been published that explore the relationship between covert narcissism and its expression in guilt and shame proneness and none has been published examining the gender differences in the expression of covert narcissism experienced by young adults in India. Thus, on this basis we propose the following hypotheses:

Hi There will be a significant relationship between Shame Proneness and covert narcissism

$\mathrm{H}_{2}$ There will be a significant relationship between Guilt Proneness and covert narcissism

$\mathrm{H}_{3}$ Shame Proneness will predict Covert Narcissism

$\mathrm{H}_{4}$ Guilt Proneness will predict Covert Narcissism

$\mathrm{H}_{5}$ There will be a significant difference in Covert Narcissism between males and females

Methodology:

Researchers agree that there are two narcissism expressions
Ambient Science, 2022: Vol. 09(1); 31-37 DOI:10.21276/ambi.2022.09.1.ta03

Overt/grandiose and Covert/ vulnerable narcissism. The existing information on narcissistic personality disorder focuses on various aspects of grandiose narcissism but very little has been explored on covert narcissism. The present research examines the experience of negative emotions such as guilt-proneness and shame proneness that impact covert narcissists' entitled perceptions of their own performance that has been overlooked. The main purpose behind conducting this study was the exploration of covert narcissism and its expression in the guilt and shame proneness experienced by young adults in India. The study aims to analyse more about the relationship between young adults' shame proneness, guilt-proneness with covert narcissism. Research also lacks to highlight gender differences in the expression of covert narcissism. As the literature suggests not many studies have focused on the gender difference and expression of negative emotions such as guilt and shame proneness and their relationship with covert narcissism in context to the Indian population in young adults.

The sample of the study consisted of 180 emerging adults. These emerging adults were selected with the following criteria of inclusion and exclusion:

\section{Criteria of Inclusion:}

1. University Students within the age group of 18 to 23 years.

2. Both Male and Female students.

3. Students residing in India

4. Students with Proficiency in the English language

Criteria of Exclusion:

1. College Dropouts or students repeating a year of the degree course.

2. Students with a physical disability or with a history of psychological illness.

In the present study, a purposive sampling technique was used to select the sample.

\section{Research Design}

A causal research design was used for the present study to investigate the relationship between the above-mentioned variables.

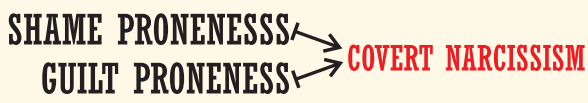

1. Maladaptive Covert Narcissism Scale, an updated version of the Hypersensitive Narcissism Scale, was developed by Cheek et al. (2013). It has 23 items measuring covert narcissism. Maladaptive Covert Narcissism Scale (MCNS) had an alpha reliability of .89 compared to .75 for the original HSNS; it correlated .65 with the MMPI measure of covert narcissism compared to .30 with the maladaptive overt narcissism factor of the NPI and -.16 with the Adaptive Overt Narcissism Scale.

2. The Guilt and Shame Proneness Scale (GASP) was developed by Taya et al. (2010). It has 16 items measuring individual differences in the propensity to experience guilt and shame across a range of personal transgressions. The GASP contains 2 guilt subscales that assess negative behaviour-evaluations (NBEs) and repair action tendencies following private 
transgressions and 2 shame subscales that assess negative self-evaluations (NSEs) and withdrawal action tendencies following publically-exposed transgressions. Alpha coefficients of .6o or higher would ensure that the reliability of the GASP is similar to the reliability of other guilt- and shame-proneness scales. It provides evidence for construct validity.

The study was conducted in two phases. In the first phase, based on the criteria of inclusion and exclusion, the study population of university students was contacted and a sample of 180 students was drawn. In the second phase, students selected in the sample were administered the tests of the study through Google Forms. Thereafter, results were compiled and trends were analyzed.

Statistical analysis was done using an IBM SPSS, including Descriptive Statistics using Mean, Standard Deviation, coefficient of correlation, Regression analysis and Independent sample T-test.

\section{Result \& Discussion}

Table 1: Means and Standard Deviations ( $\mathrm{N}=180)$

Covert Narcissism 62.5 \pm 13.0662 Shame Proneness $8.31 \pm 2.1469$ Guilt Proneness $10.28 \pm 2.4487$

Table-2: Coefficients of Correlations of Covert Narcissism (A) with Negative Behaviour Evaluation (B), Guilt Repair (C), Negative Self Evaluation (D) and Shame Withdrawal (E).

\begin{tabular}{rccccc}
\hline & $\mathrm{A}$ & $\mathrm{B}$ & $\mathrm{C}$ & $\mathrm{D}$ & $\mathrm{E}$ \\
\hline $\begin{array}{r}\text { Covert Narcissism (A) } \\
* *\end{array}$ & 1 & -.040 & -.006 & $.236^{* *} \cdot 312^{* *}$ \\
Correlation is significant at the 0.01 level (2-tailed)
\end{tabular}

A significant positive correlation between Negative Self Evaluation and Shame Withdrawal with Covert Narcissism among the emerging adults $(\mathrm{p}<0.01)$. Hence, $\mathrm{H}_{1}$ was accepted. Further, a an insignificant negative correlation between Negative Behavior Evaluation and Guilt repair with Covert Narcissism was revealed, hence, $\mathrm{H}_{2}$ was rejected.

Table- 3: Regression analysis showing prediction of Covert Narcissism by Shame Withdrawal

\begin{tabular}{|c|c|c|c|c|c|}
\hline \multicolumn{6}{|c|}{ Table-3.1: Model Summary } \\
\hline \multicolumn{6}{|c|}{ Model R R Square Adju } \\
\hline \multicolumn{6}{|c|}{ a. Predictors: (Constant), SW } \\
\hline \multicolumn{6}{|c|}{ Table- 3.2 Coefficient } \\
\hline \multirow[t]{2}{*}{ Model } & \multicolumn{2}{|c|}{ Unstandard. Coeff. } & \multicolumn{3}{|c|}{ Standard. Coeff. } \\
\hline & B & Std. Error & Beta & $\mathrm{T}$ & Sig. \\
\hline 1 (Constant) & 48.414 & 3.611 & & 13.406 & .000 \\
\hline SW & $4 \cdot 387$ & 1.004 & .312 & $4 \cdot 369$ & .000 \\
\hline
\end{tabular}

a. Dependent Variable:

Covert Narcissim

Table suggested that Shame Withdrawal contribute 9\% variance in predicting Covert Narcissism which is statistical significance $(\mathrm{p}<0.01 \& \mathrm{~F}=19.084, \beta=.312)$. Hence, $\mathrm{H}_{3}$ wasaccepted
Table-4: Regression analysis showing prediction on covert narcissism by shame Negative Self Evaluation.

\begin{tabular}{|c|c|c|c|c|}
\hline \multicolumn{5}{|c|}{ Table-4.1: Model Summary } \\
\hline Model & $\mathrm{R}$ & R Square & Adjust R square & Std. Error (estimate) \\
\hline 1 & $.236^{\mathrm{a}}$ & .055 & .050 & 13.166 \\
\hline \multicolumn{5}{|c|}{ a. Predictors: Constant) NSE } \\
\hline
\end{tabular}

Table-3.2 Coefficient

Model Unstandard. Coeff. Standard. Coeff.

\begin{tabular}{|c|c|c|c|c|c|}
\hline & & Std. Error & Beta & $\mathrm{T}$ & Sig. \\
\hline 1 Constan & 50.670 & 4.135 & & 12.254 & .000 \\
\hline NSE & 2.554 & .792 & .236 & 3.225 & .002 \\
\hline
\end{tabular}

a. Dependent Variable: Covert Narcissim

The findings suggested that Negative Self Evaluation contribute a $5 \%$ variance in predicting Covert Narcissism which is the statistical significanc ( $\mathrm{p}<0.01 \& \mathrm{~F}=10.398, \quad$ 236) Hence, $\mathrm{H}_{3}$ was accepted.

Table-5: The table shows the Independent sample t test between males and females respondents on the variable Covert Narcissism

\begin{tabular}{lllll}
\hline Male $(\mathrm{n}=56)$ & Female $(\mathrm{n}=123)$ & $\mathrm{T}$ & $\mathrm{df}$ & $\mathrm{P}$ \\
$67.77 \pm 12.904$ & $61.73 \pm 13.405$ & 2.826 & 177 & $\mathrm{P}<0.05$
\end{tabular}

The above table shows significant differences between males and females on Covert Narcissism. $(\mathrm{P}<0.05)$ hence, $\mathrm{H}_{5}$ was accepted.

The current study's findings show that distinct subtypes of shame and guilt-proneness have diverse reactions among both aspects of narcissism. The findings of the study revealed that covert narcissism and shame NSE (negative self-evaluation) have a substantial positive association. According to the findings, there is a link between vulnerable narcissism and shame withdrawal. According to the study's findings, there is a link between shame proneness (negative self-evaluation and shame withdrawal) and covert narcissism. This relationship is found to be significant at 0.01 level. This means that the higher shame indicates a higher degree of development of covert narcissistic traits. The results of the study can be supported by a study conducted by (Ghim et al., 2015) showing a positive correlation between covert narcissism and shame. Another study by Poless et al. (2018) also found that the vulnerable feature of narcissism is positively connected with shame.

The results also showed that shame withdrawal and negative self-evaluation predict and lead to covert narcissism tendencies. Feelings of shame are essential to understanding vulnerable narcissism and also interventions for at-risk young adults. Shame served as a bridge between vulnerable narcissism and a more negative self-and another model (Bilevicius et al., 2019). Another study supporting the hypothesis by Hibbard (1992) stated shame was found to be positively correlated with vulnerable 


\section{TECHNOSCIENCE ARTICLE}

style. Covert narcissism has been identified as "a pattern where shame feelings are more conscious and narcissistic feelings are dissociated" (Wright et al., 1989). Gramzow \& Tanqney (1992) from their study showed a positive association between shame and narcissism emerged when maladaptive factors were isolated. (Schie et al., 2021) concluded that more shame was reported in those young people who tended to have traits of vulnerable narcissism and struggled to be close to others. Also, a more insecure attachment style may develop when there is a discrepancy between the ideal and actual self-produced by shame experiences.

The goal of this study was to examine the relationship between covert narcissism and guilt-proneness. According to the findings, covert narcissism and guilt repair have a negative correlation. Covert narcissism and negative behaviour evaluation also have a negative relationship. This relationship was not found to be meaningful. According to similar studies, the negative association between narcissism and guilt-proneness could be explained by narcissism's lack of empathy and an excessive sense of entitlement (American Psychiatric Association, 2013). Another study in which they differentiated the two types of narcissism (overt and covert) through the disposition of shame and guilt-proneness confirmed that both the dimensions of narcissism were negatively correlated with guilt (Kingston, 2005). Another study found that narcissistic people have a strong sense of entitlement, which manifests itself as an unjustified expectation of favourable treatment. They expect to be catered to, and if this does not happen, they may become violent (American Psychiatric Association, 2013). As a result, it could be one of the reasons why they are unable to consider the wants and desires of their people. Individuals with high guilt NBE (negative behaviour evaluation) ratings are more empathetic, modest, loyal, agreeable, and generous than those with low guilt NBE scores in terms of personality. Furthermore, those with a high level of guilt NBE are more likely to want to change their behaviour and prevent future harmful behaviour (Cohen et al., 2012).

Freud (1914) originally felt that women were more narcissistic than men, based on the premise that women are more concerned with their physical appearance and are more likely to engage in self-centred behaviour "make object choices in reference to qualities desired for the self" (Wink \& Gough.,1990). However, empirical evidence suggests the opposite pattern of findings, with men having higher levels of narcissism than women (Tschanz et al., 1998). Other research, on the other hand, has revealed that the difference in narcissism between men and women is almost non-existent (Bizumic \& Duckitt., 2008). The findings from the study indicate that the average score of men on covert narcissism was significantly more than that of females. This is because males have scored higher on the
Ambient Science, 2022: Vol. 09(1); 31-37 DOI:10.21276/ambi.2022.09.1.ta03

covert narcissism variables than females. Further study by Wright et al. (1989) also revealed men to be more prone to narcissism and women to depression. Sex differences related to shame and guilt are linked to specific situations and self-images. Males have higher levels of narcissism and competitiveness as compared to females (Chan \& Cheung., 2020).

Sex differences have also been found in the relationships between hypersensitivity and the Exploitativeness/Entitlement factor of narcissism (Ryan et al., 2008) the study showed that hypersensitivity was positively correlated with the frequency of physical assault, such that more hypersensitive men actually reported greater physical assault toward their partners than their partners actually reported. In males, vulnerable narcissism was a significant positive predictor of physical/sexual abuse perpetration and in females; vulnerable narcissism emerged as a significant positive predictor of physical/sexual and psychological abuse perpetration (Valashjardi et al., 2020). The gender difference in narcissism is crucial to highlight since it implies actual disparities in the latent characteristic, driven by men's heightened sense of entitlement and authority. The etiology of covert narcissism must be studied further, as well as the effect of gender stereotypes on the emergence of covert narcissistic features.

Finally, this study aimed at exploring the relationship of guilt-proneness and shame proneness with covert narcissism and its expression in young adults in India. The sample consisted of 180 young adults from the age range of 18-23 years. Findings from the current study indicate that shame proneness significantly positively relates to covert narcissism and predicts covert narcissism. It further concludes that males have significantly higher tendencies of the development of covert narcissism as compared to females.

\section{Implication:}

- The present study will serve as useful literature both for the academic and students for insight in the understanding of covert narcissism.

- It'll help in throwing more light on the covert and vulnerable types of narcissism.

- It will also help in devising early intervention programmes to eliminate shame and guilt to lessen its impact on the development of maladaptive personality patterns.

\section{Limitations of the study}

- In the following study Questionnaire as the tool is used for data collection and it is prone to have a socially desirable response.

- Non responded Questions affect the results of the study.

- Therewas a low participation rate

- Lesser literature was available in context to Covert Narcissism.

- Different perceptions of the items in the study could have led to subjectivity in the results.

- Quantitative measures of data collection were used. 
Qualitative measures like an interview, case study method was not used for data collection.

- The study was conducted during the COVID-19 pandemic which affected the availability of the responses.

\section{Futuredirections}

- It can be explored in other age groups, specifically adolescents in their developmental age.

It can be conducted on a larger sample to generalize the results.

- Other types of narcissism and their relationship with guilt and shame proneness can be studied.

- Other variables in context to clinical psychology can be explored with covert narcissism to get deeper insights.

\section{References:}

Adler, A. \& Wolfe, W.B. (1954): Understanding Human Nature. Pub. by: Fawcett Publications, New York. P. 224.

American Psychiatric Association (2013): The Diagnostic and Statistical Manual of Mental Disorders (5th ed.).Pub. by: American Psychiatric Association, Washington DC.

Andrews, B. (1998): Methodological and definitional issues in shame research, pp. 39-54. In: P. Gilbert \& B. Andrews (Eds.), Shame: Interpersonal Behavior, Psychopathology and Culture. Pub. by: Oxford University Press, New York.

Besser, A. \& Priel, B. (2008). Emotional responses to a romantic partner's imaginary rejection: The roles of attachment anxiety, covert narcissism, and self-evaluation. J. Personal., 77:287-325.

Bilevicius, E., Neufeld, D.C., Single, A., Foot, M., Ellery, M., Keough, M.T. \& Johnson, E.A. (2019): Vulnerable narcissism and addiction: the mediating role of shame. Addict. Behav., 92:115121.

Bizumic, B. \& Duckitt, J. (2008): My group is not worthy of me: Narcissism and ethnocentrism. Political Psychol., 29(3):437453 .

Cain, N.M., Pincus, A.L. \& Ansell, E.B. (2008): Narcissism at the crossroads: Phenotypic description of pathological narcissism across clinical theory, social/personality psychology, and psychiatric diagnosis. Clinic. Psychol. Rev., 28(4):638-656.

Campbell, W.K. \& Foster, C.A. (2002): Narcissism and commitment in romantic relationships: an investment model analysis. Personal. Soc. Psychol. Bull., 28(4), 484-495.

Chan, C.Y. \& Cheung, K.L. (2020). Exploring the gender differences in relationships between narcissism, competitiveness, and mental health problems among college students. J. Am. College Health, 16:1-10.

Cheek, J.M., Hendin, H.M. \& Wink., P.M. (2013): An extended version of the Hypersensitive Narcissism Scale (The Maladaptive Covert Narcissism Scale). Presented at: Association for Research in Personality, Charlotte NC

Cohen, T.R., Panter, A.T. \& Turan, N. (2012): Guilt proneness and moral character. Current Direct. Psychol. Sci., 21(5):355-359.

Cohen, T.R., Wolf, S.T., Panter, A.T. \& Insko, C.A. (2011): Introducing the GASP scale: a new measure of guilt and shame proneness. $J$. Person. Soc. Psychol., 100(5):947-966.

Foster, J.D., \& Trimm, R.F. VI (2008): On being eager and uninhibited: Narcissism and approach-avoidance motivation. Personal. Soc. Psychol. Bull., 34(7):1004-1017.
Ghim, S.C., Choi, D.H., Lim, J.J. \& Lim, S.M. (2015): The relationship between covert narcissism and relational aggression in adolescents: Mediating effects of internalized shame and anger rumination. Int. J. Info. Edu. Technol., 5(1):21.

Gilbert, P. (1997): The evolution of social attractiveness and its role in shame, humiliation, guilt and therapy. $\mathrm{Br}$. J. Med. Psychol., 70:113-147.

Gramzow, R. \& Tangney, J.P. (1992): Proneness to shame and the narcissistic personality. Personal. Soc. Psychol. Bull., 18(3):369-376.

Harder, D.W. \& Greenwald, D.F. (1999): Further validation of the shame and guilt scales of the Harder Personal Feelings Questionnaire-2. Psychol. Rep., 85(1):271-281.

Hendin, H.M. \& Cheek, J.M. (1997): Assessing hypersensitive narcissism: A reexamination of Murray's Narcism Scale. J. Res. Personal., 31(4):588-599.

Kaufman, G. (1989): The Psychology of Shame: Theory and Treatment of Shame-based Syndromes. Pub. by: Springer, New York.

Kealy, D. \& Rasmussen, B. (2012): Veiled and vulnerable: the other side of grandiose narcissism. Clinical Soc. Work I., 40(3):356365.

Kingston, P. (2005): Differentiating the two types of narcissism through the disposition to shame and guilt-proneness. Dissertation Abstracts International: Section B: The Sciences and Engineering, 66(1-B):558.

Krizan, Z. \& Bushman, B.J. (2011): Better than my loved ones: Social comparison tendencies among narcissists. Personal. Indiv. Differ., 50(2):212-216.

Krizan, Z. \& Herlache, A.D. (2017): The narcissism spectrum model: A synthetic view of narcissistic personality. Personal. Soc. Psychol. Revi., 22(1):108886831668501

Krizan, Z. \& Johar, O. (2012): Envy divides the two faces of narcissism. L.Personal., 8o(5):1415-1451.

Levin, S. (1967): Some metapsychological considerations on the differentiation between shame and guilt. Int. J. Psychoanal., 48(2): 267-276.

Lukowitsky, M.R. \& Pincus, A.L. (2013): Interpersonal perception of pathological narcissism: a social relations analysis. J. Personal. Assess., 95(3):261-273.

Miller, J.D. \& Maples, J. (2011): Trait personality models of narcissistic personality disorder, grandiose narcissism, and vulnerable narcissism, pp. 71-88. In: W. K. Campbell \& J. D. Miller (Eds.), The Handbook of Narcissism and Narcissistic Personality Disorder: Theoretical Approaches, Empirical Findings, and Treatments. Pub. by: John Wiley \& Sons, Inc.

Peters, J.R. \& Geiger, P.J. (2016): Borderline personality disorder and self-conscious affect: Too much shame but not enough guilt? Personal. Disor.: Theo. Res. Treat., 7(3):303-308.

Piers, G. \& Singer, M.B. (1953): Shame and guilt; a psychoanalytic and a cultural study. Charles $C$. Thomas.

Pincus, A.L. \& Roche, M.J. (2011): Narcissistic grandiosity and narcissistic vulnerability, pp. 31-40. In: W.K. Campbell \& J.D. Miller (Eds.), The Handbook of Narcissism and Narcissistic Personality Disorder: Theoretical Approaches, Empirical Findings, and Treatments. Pub. by: 


\section{TECHNOSCIENCE ARTICLE}

Wiley, Singapore.

Pincus, A.L., Ansell, E.B., Pimentel, C.A., Cain, N.M., Wright, A.G.C. \& Levy, K.N. (2009): Initial construction and validation of the Pathological Narcissism Inventory. Psychol. Assess., 21:365-379.

Poless, P. G., Torstveit, L., Lugo, R. G., Andreassen, M. \& Sütterlin, S. (2018): Guilt and proneness to shame: unethical behaviour in vulnerable and grandiose narcissism. Eu. J. Psychol., 14(1):28-43.

Ritter, K. (2014): Shame in patients with a narcissistic personality disorder. Psych. Res., 215(2):429-437.

Rose, P. (2002): The happy and unhappy faces of narcissism. Person. Indiv. Differ., 33:379-391.

Ryan, K.M., Weikel, K. \& Sprechini, G. (2008): Gender differences in narcissism and courtship violence in dating couples. Sex Roles, 58(11-12):802-813.

Schie, C.C.V., Jarman, H.L., Reis, S. \& Grenyer, B.F. (2021): Narcissistic traits in young people and how experiencing shame relates to current attachment challenges. $B M C P$ sych., 21:246.

Schurman, C.L. (200o): Social phobia, shame and hypersensitive narcissism. Dissert. Abs. Int., 61(09):5004.
Ambient Science, 2022: Vol. 09(1); 31-37 DOI:10.21276/ambi.2022.09.1.ta03

Shapiro, L.J., \& Stewart, E.S. (2011): Pathological guilt: a persistent yet overlooked treatment factor in obsessive-compulsive disorder. Ann Clin Psychiatry., 23(1):63-70.

Tschanz, B.T., Morf, C.C. \& Turner, C.W. (1998). Gender differences in the structure of narcissism: A multi-sample analysis of the Narcissistic Personality Inventory. Sex Roles, 38(9-10):863-870.

Valashjardi, A., MacLean , R. \& Charles , K. (2020). Unmasking gender differences in narcissism within intimate partner violence. Person. Indiv. Differ., 167:270-285.

Wink, P. \& Gough, H.G. (1990): New narcissism scales for the California psychological Inventory and MMPI._J. Person. Assess., 54(3-4):446-462.

Wink, P. (1991): Two faces of narcissism. J. Person. Soc. Psychol., 61(4):590-597.

Wright, F., O'Leary, J. \& Balkin, J. (1989): Shame, guilt, narcissism, and depression: Correlates and sex differences. Psychoan. Psychol., 6(2):217-230. 